\title{
Artigo original \\ Nível de atividade física como preditor de fatores de risco cardiovasculares em crianças
}

\author{
Alynne Christian Ribeiro Andaki \\ Adelson Luiz Araújo Tinoco \\ Universidade Federal de Viçosa \\ Roberto Andaki Júnior \\ Amanda Santos \\ Universidade Federal do Triângulo Mineiro \\ Ciro José Brito \\ Universidade Federal de Sergipe \\ Edmar Lacerda Mendes \\ Universidade Federal do Triângulo Mineiro
}

\begin{abstract}
Resumo-O presente estudo avaliou a eficácia do nível de atividade física (NAF), envolvimento em atividade física de moderada a vigorosa intensidade (AFMV) e número de passos/dia para predição dos fatores de risco cardiovasculares (FRCV) em crianças. Foram mensuradas medidas antropométricas, composição corporal, pressão arterial, glicemia, HDL-c e triacilglicerídeos. NAF e envolvimento em AFMV foram obtidos pelo registro de atividade física e número de passos por pedômetro. Participaram 187 crianças com 9,90 \pm 0,7 anos de idade. NAF apresentou área sob a curva ROC significativa para predição da obesidade, alto \% de gordura corporal e pressão arterial alterada (PAA) nas meninas. NAF foi acurado em predizer obesidade e síndrome metabólica (SM) e AFMV foi acurada em predizer SM e PAA nos meninos. Meninos que atingiram a recomendação de 13000 passos/dia foram protegidos da SM e baixo HDL-c. Atender as recomendações de envolvimento em AFMV e número de passos protegem crianças dos FRCV.
\end{abstract}

Palavras-chaves: obesidade, criança, doenças cardiovasculares

Abstract- - Physical activity level as a predictor of cardiovascular risk factors in children.” This study evaluated the effectiveness of the physical activity level (PAL), engagement in physical activity of moderate to vigorous intensity (MVPA) and number of steps/day as prediction of cardiovascular risk factors (CVRF) in children. We measured anthropometric parameters, body composition, blood pressure, blood glucose, HDL-c and triglycerides. The PAL and engagement in MVPA were obtained using a questionnaire and computing number steps/days with a pedometer. Participants were 187 children, with a mean age of $9.90( \pm 0.7)$ years. The PAL showed a significant area under the ROC curve as a predictir of obesity, high body fat \%, and high blood pressure (HBP) in girls. The PAL accurately predicted obesity and metabolic syndrome (MS), and MVPA accurately predicted MS and HBP in boys. Boys who achieved the recommended 13.000 steps/day were protected from MS and low HDL-c. Achieving the recommendations of engagement in MVPA and number of steps were protection against CVRFfactors in children.

Keywords: obesity, children, cardiovascular diseases

Resumen- - El nivel de actividad física como un predictor de riesgo cardiovascular en niños.” En este estudio se evaluó la efectividad del nivel de actividad física (NAF), la participación en la actividad física de intensidad moderada a vigorosa (ATMV) y el número de pasos / día para la predicción de los factores de riesgo cardiovascular (FRCV) en niños. Medimos las medidas antropométricas, composición corporal, presión arterial, glucemia, colesterol HDL y triglicéridos. NAF y la participación en ATMV se obtuvieron los registros de la actividad física y los pasos podómetro por número. 187 niños participan 9,90 \pm 0,7 años. NAF tuvo un área bajo la curva ROC significativa para la predicción de la obesidad, alto\% de grasa corporal y la presión arterial enmendada (PAE) en las niñas. NAF fue precisa en la predicción de la obesidad y el síndrome metabólico (SM) y moderada a vigorosa fue precisa en la predicción de SM y PAE en los niños. Los niños que alcanzaron la cantidad recomendada de 13000 pasos /día fueron protegidos de la SM y bajo HDL-c. Alcanzar las recomendaciones de la participación en ATMV y el número de pasos proteger a los niños de los FRCV.

Palabras claves: obesidad, niños, enfermedades cardiovasculares 


\section{Introdução}

Doenças cardiovasculares representam a maior causa de morte no Brasil (Ministério da Saúde, 2006) e, embora se manifeste clinicamente na idade adulta, os fatores de risco podem surgir ainda na infância (Freedman et al., 2008). Atividade física (AF) é conceitualmente entendida como qualquer movimento corporal produzido pelos músculos esqueléticos que resulta em gasto energético (Caspersen, Powell, \& Christenson, 1985). Diretrizes de saúde pública sobre a prática de AF com objetivo de promover benefícios à saúde baseiam-se suas recomendações expressas em frequência, duração e intensidade. Benefícios da prática regular de AF para saúde são reconhecidos e amplamente difundidos pela comunidade científica. Ademais, o baixo nível de atividade física (NAF) está associado ao risco de doenças cardiovasculares (Andersen et al., 2008; Froberg \& Andersen, 2005).

Crianças e adolescentes devem acumular 60 minutos/dia de AF de moderada a vigorosa intensidade e, de duas ou três vezes por semana incluir exercícios de flexibilidade, fortalecimento muscular e ósseo (Department of Health, 2004; U.S. Department of Health and Human Services, 2008; World Health Organization, 2010).

Dentre as medidas utilizadas para estimar o gasto energético e níveis de atividade física, o equivalente metabólico da tarefa (METs) é largamente utilizado (Pulsford et al., 2011; Tremblay et al., 2011). AF leve envolve gasto energético entre 1,6 e 2,9 METs (Pate, O'Neill, \& Lobelo, 2008). Atividades de intensidade moderada variam entre 3,0 e 6,0 METs e atividade física vigorosa acima de 6,0 METs (Haskell et al., 2007; Tudor-Locke, Washington, Ainsworth, \& Troiano, 2009). O número de passos/dia dados pelos usuários registrados por pedômetros fornecem volume de atividade física diário aproximado e são mais propensos a serem adotados devido à facilidade de interpretação e relativo baixo custo (Tudor-Locke et al., 2011). A utilização do NAF, tempo despendido em AF de moderada a vigorosa intensidade e número de passos/dia podem ser considerados ferramentas importantes para determinar o risco de desenvolvimento de doença cardiovascular. Assim, são necessários valores de referência de forma a facilitar recomendações da prática de AF para benefícios à saúde em crianças.

Contudo, permanecem escassos, principalmente na população infantil, estudos sobre pontos de corte para medidas da AF associados ao risco de doenças cardiovasculares. Assim, o presente estudo avaliou a eficácia do NAF, envolvimento em AF de moderada a vigorosa intensidade e número de passos/dia na predição dos fatores de risco cardiovasculares (FRCV) em crianças do município de Viçosa, MG.

\section{Método}

\section{Delineamento do estudo e casuística}

Trata-se de estudo epidemiológico observacional, de delineamento transversal, amostra probabilística, com participação de 187 escolares, de ambos os sexos, matriculados no $4^{\circ}$ e $5^{\circ}$ ano do ensino fundamental do município de Viçosa, MG e que atenderam aos seguintes critérios: a) não faziam uso de medicamento; b) não estavam em dieta de restrição calórica; c) foram autorizados pelos pais ou responsáveis; d) respeitaram o jejum de 12 horas para a coleta de sangue.

Para o cálculo do tamanho mínimo amostral, utilizou-se a equação [n = P x Q / (E/1,96)² (Lwanga \& Lemeshow, 1991)], em que n equivale ao tamanho mínimo da amostra necessária; P equivale a taxa máxima de prevalência (50\%); Q é igual a 100-P; e E é a margem de erro amostral tolerado. Considerando o número total de escolares no $4^{\circ}$ e $5^{\circ}$ ano do ensino fundamental, número total da população, erro tolerável de $5 \%$ e nível de confiança de $95 \%$, para comprovação probabilística foi necessário amostra mínima de 107 crianças.

\section{Procedimentos}

Após a aprovação do estudo pelo Comitê de Ética em Pesquisa com Seres Humanos da Universidade Federal de Viçosa (Of. Ref. 060/2009) e autorização dos pais ou responsáveis, as crianças foram submetidas a avaliação antropométrica, composição corporal e aferição da pressão arterial. As crianças foram instruídas ao correto preenchimento do registro de atividades físicas. Em seguida, os participantes foram convidados a se deslocarem ao laboratório de análises clínicas para a coleta de sangue. $\mathrm{Na}$ última etapa as crianças receberam o pedômetro (contador de passos) que fora utilizado durante sete dias consecutivos.

\section{Antropometria e composição corporal}

A massa corporal foi obtida por balança eletrônica digital (Plena, Lumina) com capacidade máxima de 150 kg e precisão de 100 g, conforme técnicas padronizadas (WHO, 1995). A estatura foi obtida por meio de um estadiômetro portátil (Rigor e Técnica) com comprimento de $2 \mathrm{~m}$ e escala de $0,1 \mathrm{~cm}$ seguindo normas padronizadas (WHO, 1995). O índice de massa corporal (IMC) foi calculado com as medidas de peso e estatura por meio da fórmula: IMC = Peso (kg)/estatura $\left(\mathrm{m}^{2}\right)$, e as crianças foram classificadas com sobrepeso e obesidade, segundo os critérios estabelecidos por Cole, Bellizzi, Flegal e Dietz (2000).

O perímetro da cintura foi obtido ao final do movimento expiratório normal com fita métrica flexível e inelástica com extensão de 2m (Sanny). Todas as medidas foram feitas em triplicata, considerando-se para análise o valor médio entre elas. Foram mensurados os seguintes pontos anatômicos: menor curvatura do abdome, entre a crista-ilíaca e as costelas (Lohman, Roche, \& Martorell, 1988); ponto médio entre a crista-ilíaca e a última costela (WHO, 2000) e sobre a cicatriz umbilical.

Foram obtidas as dobras cutâneas tricipital (DCT), bicipital (DCB), subescapular (DCSe) e supra-ilíaca (DCSi), utilizando-se o adipômetro Lange Skinfold Caliper (Guedes 
\& Guedes, 2006). Os resultados foram interpretados isoladamente, bem como pelo somatório da espessura de duas dobras (DCT+ DCSe) e das quatro dobras cutâneas avaliadas, com os resultados expressos em milímetros (mm). Para o cálculo do percentual de gordura corporal (\%GC), foram utilizadas as equações propostas por Slaughter et al. (1988). A partir dos valores de \% GC, a amostra foi classificada de acordo com as categorias de adiposidade propostas por Lohman (1987).

\section{Pressão arterial}

A pressão arterial foi aferida com esfigmomanômetro de coluna de mercúrio com manguitos de tamanhos apropriados a circunferência dos braços das crianças seguindo as normas propostas pelas VI Diretrizes Brasileiras de Hipertensão (Nobre, 2010). Após repouso aproximado de 5 a 10 minutos, as crianças sentadas tiveram sua pressão aferida por três vezes com um intervalo de no mínimo 1 min entre as aferições e considerou-se o valor médio das duas últimas aferições.

A classificação da pressão arterial foi determinada com base nos pontos de corte específicos para crianças, de acordo com o percentil para estatura e sexo (Nobre, 2010). Aqueles voluntários que apresentaram valores pressóricos elevados tiveram sua pressão arterial aferida em outros dois momentos distintos e receberam recomendação para avaliação médica. Só foram diagnosticadas com pressão arterial elevada as crianças que apresentaram a pressão arterial sistólica ou diastólica acima do percentil 90, em três dias distintos em que foram feitas as aferições.

\section{Nível de atividade física}

Para estimar o envolvimento em AF de moderada a vigorosa intensidade foi utilizado o registro recordatório de Bouchard et al. (1983). Os dados obtidos foram coletados em dois dias de semana e um dia de final de semana. O nível de AF foi calculado pela razão do gasto energético diário total pela taxa metabólica basal (NAF = GET/TMB). O GET foi obtido pelo recordatório de atividades, média dos três dias coletados, e para a estimativa da taxa metabólica basal utilizou-se a fórmula World Health Organization (1985) para crianças de 3 a 10 anos de idade.

Foi também realizado monitoração por sensor de movimento. As crianças utilizaram um pedômetro (Pulse Rate Pedometer, modelo DX 8897) e foram orientadas quanto a utilização e funcionamento do aparelho. Receberam ficha específica em que faziam as anotações do número de passos dados durante o período avaliado. Uma das recomendações para crianças nesta faixa etária são 11.000 passos/dia para meninas e 13.000 passos/dia para meninos (Vincenti \& Pangrazi, 2002). Para melhor reprodutibilidade, os valores utilizados foram a média do número de passos dados por dia, durante os sete dias consecutivos.

\section{Avaliação bioquímica}

Amostras sanguíneas foram coletadas individualmente após jejum de 12 a 14 horas. Amostras de sangue (5 mL) foram coletadas em tubos a vácuo BD Vacutainer ${ }^{\circledR}$ e centrifugadas a $3400 \mathrm{rpm}$ durante oito minutos para separar o soro dos demais componentes do sangue. Amostras do soro foram analisadas para a determinação do HDL colesterol, triglicerídeos e glicemia com auxílio de analisador hematológico automatizado (Cobas Mira Plus- Roche). Para a determinação quantitativa do HDL-c foi utilizado o método enzimático colorimétrico homogêneo com kit Colesterol HDL Direto SP (Human do Brasil) CV \% = 0,73. Para dosagem dos triglicerídeos foi utilizado o kit Triglicérides Liquicolor Mono, CV\% = 1,65 (Human do Brasil) empregando o método enzimático-colorimétrico com fator clareante de lípides. Já a glicemia foi medida pelo método enzimático colorimétrico, utilizado-se o kit Glicose Liquicolor, CV \% = 0,6 (Human do Brasil).

\section{Critérios para diagnóstico da síndrome metabólica}

A definição da SM dada pelo National Cholesterol Education Programs - Adult Treatment Panel III (NCEP, 2001) consiste na presença de pelo menos três dos seguintes fatores: obesidade (caracterizada pela obesidade abdominal), dislipidemia (altos níveis de triglicerídeos ou baixo nível de HDL-c), hipertensão arterial e hiperglicemia de jejum. O consenso sobre a SM para crianças ainda é ausente. Para o diagnóstico da SM no presente estudo, as crianças deveriam apresentar no mínimo três dos seguintes fatores: triglicerídeos $\geq 100 \mathrm{mg} / \mathrm{dL}, \mathrm{HDL}$ - c $<50 \mathrm{mg} / \mathrm{dL}$, glicemia $\geq$ $110 \mathrm{mg} / \mathrm{dL}$, circunferência de cintura $\geq$ percentil 75 para idade e sexo, e alteração na pressão arterial (diastólica ou sistólica) > percentil 90 ajustado para idade, estatura e sexo. Optou-se pelos pontos de cortes adaptados a idade da população estudada segundo os critérios de Ferranti et al. (2004).

\section{Análise estatística}

A estruturação do banco de dados foi realizada no software Epidata versão 3.1. Foram utilizados para as análises estatísticas os softwares SPSS 17.0 e Medcalc 11.0.1. Todas as variáveis foram testadas quanto a sua normalidade pelo teste de Kolmogorov-Smirnov. A análise descritiva apresentou as variáveis contínuas em média e desvio padrão; e as categóricas em valores percentuais. O teste t de Student foi utilizado para comparação entre as médias de grupos independentes com distribuição paramétrica e Mann-Whitney para as amostras com distribuição não-paramétrica. A análise de variância (ANOVA) foi utilizada para comparação entre três ou mais grupos independentes, seguida pelo post-hoc de Bonferroni. O teste de Qui-quadrado foi utilizado para verificar heterogeneidade entre as proporções. O nível de significância adotado foi de á = 5 \%. Foram sugeridos pontos de corte para as variáveis antropométricas por meio das 
curvas ROC (Receiver Operating Characteristic). A significância estatística de cada análise foi verificada pela área sob a curva ROC (AUC) e pelo limite inferior do intervalo de confiança (IC) a $95 \%$ maior que 0,5 (Schisterman, Faraggi, Reiser, \& Trevisan, 2001).

Foram considerados FRCV as variáveis de desfecho do estudo: obesidade, alto percentual de gordura corporal, hipertrigliceridemia, reduzidos níveis de HDL-c, pressão arterial alterada e síndrome metabólica.

\section{Resultados}

Participaram do estudo 187 crianças, de ambos os sexos, com idade média de 9,9 $\pm 0,7$ anos. O FRCV de maior prevalência na amostra foi o excesso de gordura corporal (39,6\%), seguido pelo baixo nível de HDL-c $(24,1 \%)$, sobrepeso e obesidade (20,9\%), pressão arterial alterada (14,4\%), hipertrigliceridemia (10,6\%), e síndrome metabólica $(8,5 \%)$. Não houve diferença significativa entre as proporções de FRCV entre meninos e meninas $(p>0,05)$, Tabela 1 .

O NAF (GET/TMB) e o número de passos, não diferiram significativamente entre os sexos $(p>0,05)$. Apenas $13,6 \%$ das meninas e $14,5 \%$ dos meninos alcançaram a recomendação de 11.000 e 13.000 passos/dia, respecti-vamente. A proporção de meninos que atingiram a recomendação de envolvimento em pelo menos 60 mim diários em AF de moderada a vigorosa intensidade foi significativamente superior a das meninas ( $78 \%$ vs 55,3\%, $p=0,02$ ), respectivamente. $\mathrm{O}$ tempo de envolvimento em AF de moderada a vigorosa intensidade foi significativamente superior durante um dia de final de semana do que durante os dias de semana $\left[\mathrm{F}_{2,239}=\right.$ 11,504; $p=0,001$ ] (Figura 1).

O NAF foi acurado em predizer alto \% GC (NAF > 2,7), sobrepeso e obesidade e PAA (NAF $>3,04)$ para meninas ( $p$ $<0,05)$, Tabela 2 .

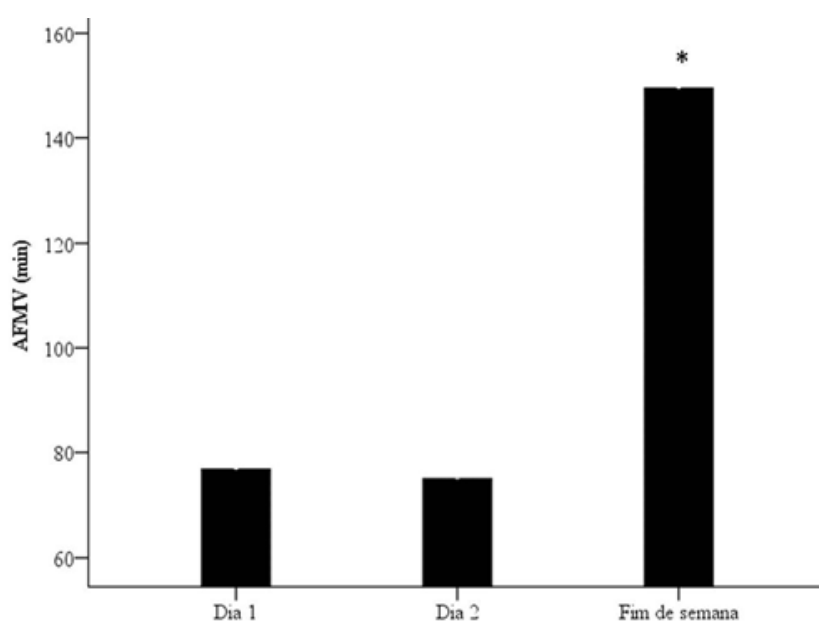

Figura 1. Tempo de envolvimento em atividade física de moderada a vigorosa intensidade durante dois dias de semana e um de final de semana.
Tabela 1. Distribuição dos fatores de risco cardiovasculares em crianças, escolares do município de Viçosa, MG.

\begin{tabular}{lccccc}
\hline \multirow{2}{*}{ FRCV } & \multicolumn{3}{l}{ Meninas } & \multicolumn{2}{c}{ Meninos } \\
\cline { 2 - 5 } & $\mathrm{n}$ & $\%$ & $\mathrm{n}$ & $\%$ & \\
& & & & & \\
\hline Sobrepalor \\
Alto \% GC & 23 & 21,7 & 16 & 19,8 & 0,8 \\
Hipertrigliceridemia & 12 & 14,1 & 3 & 5,4 & 0,1 \\
Reduzido HDL-c & 20 & 23,5 & 14 & 25 & 0,1 \\
Pressão Arterial Alterada & 16 & 15,1 & 11 & 13,6 & 0,9 \\
Síndrome Metabólica & 9 & 10,5 & 3 & 5,3 & 0,4 \\
\hline
\end{tabular}

Alto \% GC = alto percentual de gordura corporal; $\mathrm{FRCV}=$ fatores de risco cardiovasculares

Entre os meninos o NAF foi acurado em predizer reduzidos níveis de HDL-c e SM (NAF > 3,55), alto \% GC $(\mathrm{NAF}>2,80)$ e sobrepeso e obesidade (NAF $>3,25)(p<0,05)$. Ademais, observou-se que meninos que atingiram a recomendação de 13000 passos/dia estão protegidos da SM e baixo HDL-c $(p<0,05)$. A proteção para SM e PAA foi determinada pelos pontos de corte de envolvimento em AF de moderada a vigorosa intensidade acima $155 \mathrm{~min}$ e $135 \mathrm{~min}$, respectivamente.

\section{Discussão}

A promoção da atividade física na infância tem sido apontada como intervenção ideal para evitar o desenvolvimento de doenças cardiovasculares ao longo da vida (Steinberger \& Daniels, 2003). O presente estudo apresenta uma proposta simples, de fácil aplicabilidade, reprodutibilidade, e baixo custo para predizer o risco de desenvolver doenças cardiovasculares em crianças, contribuindo, assim, com as discussões sobre os pontos de corte para o nível de atividade física, número de passos/dia e envolvimento em AF de moderada a vigorosa intensidade como preditor de fatores de risco cardiovasculares em crianças.

Dentre as evidências obtidas, observou-se que, na amostra avaliada, a gordura corporal é o principal determinante de FRCV. Não houve diferença entre gêneros quanto ao nível de atividade física, sendo o tempo de atividade física maior aos finais de semana. Nas meninas o nível de atividade foi acurado preditor para sobrepeso e obesidade, excesso de gordura corporal e pressão arterial alterada. Nos meninos, foi predidor para o HDL-c, SM, excesso de gordura corporal, sobrepeso e obesidade.

Analisando a amostra como um todo, o excesso de gordura corporal e baixo nível de HDL-c foram os FRCV mais 
Tabela 2. Predição de fatores de risco cardiovasculares, em meninas por meio do NAF.

\begin{tabular}{lllllll}
\hline FRCV & AUC & IC 95\% & $p$-valor & PC & S & E \\
\hline Sobrepeso e Obesidade & 0,976 & $0,880-0,989$ & $<0,001$ & $>3,04$ & 90 & 91 \\
Alto \%GC & 0,841 & $0,740-0,915$ & $<0,001$ & $>2,70$ & 83 & 78 \\
PAA & 0,719 & $0,604-0,816$ & 0,004 & $>3,04$ & 67 & 76
\end{tabular}

FRCV = fatores de risco cardiovasculares; AUC = área sob a curva ROC; $\mathrm{PC}$ = ponto de corte; $\mathrm{S}=$ sensibilidade, $\mathrm{E}=$ especificidade; Alto \%GC= alto percentual de gordura corporal; PAA = pressão arterial alterada.

Tabela 3.Predição de fatores de risco cardiovasculares em meninos, por meio de NAF, número de passos e minutos de envolvimento em atividade física de moderada a vigorosa intensidade.

\begin{tabular}{lllllll}
\hline FRCV & AUC & IC 95\% & p-valor & PC & S & E \\
\hline NAF & & & & & & \\
HDL-c & 0,883 & $0,681-0,978$ & 0,001 & $>3,55$ & 100 & 75 \\
Alto \%GC & 0,789 & $0,632-0,902$ & 0,001 & $>2,80$ & 84 & 62 \\
Sobrepeso e Obesidade & 0,969 & $0,859-0,999$ & 0,001 & $>3,25$ & 100 & 75 \\
SM & 0,817 & $0,601-0,945$ & 0,001 & $>3,55$ & 100 & 75 \\
Número de passos & & & & & \\
Alto \%GC & 0,657 & $0,511-0,785$ & 0,04 & $>6686$ & 79 & 52 \\
HDL-c & 0,817 & $0,647-0,928$ & 0,03 & $>9657$ & 67 & 97 \\
SM & 0,891 & $0,736-0,971$ & 0,001 & $>7872$ & 100 & 78 \\
Envolvimento em AFMV (min) & & & & & & \\
SM & 0,810 & $0,599-0,939$ & 0,001 & 155 & 100 & 76 \\
PAA & 0,769 & $0,612-0,886$ & 0,001 & 135 & 100 & 72,2 \\
\hline
\end{tabular}

FRCV $=$ fatores de risco cardiovasculares; AUC = área sob a curva ROC; $\mathrm{S}=$ sensibilidade, $\mathrm{E}$ = especificidade; Alto \%GC= alto percentual de gordura corporal; PAA = pressão arterial alterada; AFMV = atividade física de moderada a vigorosa intensidade.

prevalentes. Resultados similares foram observados por Weghuber et al. (2013) onde maiores níveis de adiposidade corporal foram diretamente associados a risco metabólico em adolescentes. Baixos níveis de HDL-c são perigosos para a saúde cardiovascular de crianças, Schusterova, Leenen, Jurko, Sabol, e Takacova (2013) observaram que crianças ( 13 anos) com o IMC > percentil 85 apresentam significativamente menor concentração de HDL-c, ademais, este mesmo estudo evidenciou que os níveis de HDL-c apresentam correlação inversa $(r=-0,5)$ com os níveis de tecido adiposo no epicárdio. Apesar de pouco estudado em crianças, o nível de adiposidade no epicárdio é considerado ótimo preditor do risco cardiovasculares em adultos (Lacobellis, Willens, Barbaro, \& Sharma, 2008).

O NAF foi preditor de sobrepeso e obesidade em ambos os sexos. A prática de AF de moderada a vigorosa intensidade parece ser significativamente maior em crianças não-obesas (3,9\% do tempo monitorado, equivalente a $23 \mathrm{~min} / \mathrm{dia}$ ) do 
que obesas (2,4\% do tempo monitorado, equivalente a 16 min/dia) (Hughes et al., 2006). Estes ainda apresentam diferenças significativas quanto a fatores de risco cardiovasculares (HDL-c, triglicerídeos, HOMA-IR), com perfil lipídico deletério encontrado em crianças obesas (Danielsen et al., 2011). Praticar pelo menos 1 h/dia de AF de moderada a vigorosa intensidade tem sido associado com diminuição de $1,2 \mathrm{~kg} / \mathrm{m}^{2}$ do IMC e 3,2 cm de perímetro da cintura (Colley et al., 2012).

As respostas sobre níveis de atividade física entre gêneros modificam entre os diferentes levantamentos, em adolescentes do sul do Brasil, houve maior prevalência de meninas ativas (Farias Júnior et al., 2009), por outro lado, levantamentos realizados na China (Kong et al., 2010; Li, Dibley, Sibbritt, \& Yan, 2006), Austrália (Hume et al., 2012), Reino Unido (Pearce, Basterfield, Mann, Parkinson, \& Adamson, 2012) e Estados Unidos da América (Anderson, Economos, \& Must, 2008; Eaton et al., 2010) indicaram maior prevalência de meninos ativos. Segundo evidências apresentadas por Adams, Caparosa, Thompson, e Norman (2009), adolescentes com sobrepeso devem caminhar pelo menos 10.000 passos por dia para alcançar 60 min de AF de moderada a vigorosa intensidade por dia. Em nosso estudo, a medida realizada por pedômetro não foi condizente a alta prevalência de crianças que alcançaram o mínimo recomendado de AF de moderada a vigorosa intensidade pelo recordatório de Bouchard. Resultado que confirmam os achados da revisão sistemática de Adamo, Prince, Tricco, Connor-Gorber, e Tremblay (2009) onde 72 \% dos estudos sobre medida direta (acelerômetro) e indireta (questionário) da atividade física em crianças apresentam resultados superestimados quando a medida é realizada indiretamente. Como em nossos achados, estudos têm encontrado baixos NAF entre crianças e adolescentes (King et al., 2010; Pearce et al., 2012) e, se combinado ao comportamento sedentário excessivo (Anderson et al., 2008; Harrison, Burns, McGuinness, Heslin, \& Murphy, 2006; Mushtaq et al., 2011; Sisson, Broyles, Baker, \& Katzmarzyk, 2010) agrava riscos de excesso de peso e surgimento de doenças cardiovascular. Sendo assim, para predizer os FRCV, estudos defendem o envolvimento em AF de moderada a vigorosa intensidade acima dos $60 \mathrm{~min} / \mathrm{dia}$ recomendados por diretrizes internacionais (Activity level and cardiovascular risk, 2006; Andersen et al., 2008).

Cabe destacar que a quantidade de passos mínimos diários varia entre estudos (Adams et al., 2009). A recomendação americana de 13.000 passos/dia para o sexo masculino (C. Tudor-Locke, \& Bassett, 2004), foi alcançada por 14,5 \% dos meninos. O ponto de corte encontrado no presente estudo, que mais se aproximou desta recomendação foi $12.759 \mathrm{passos} / \mathrm{dia}(\mathrm{S}=50 \% ; \mathrm{E}=100 \%$ ). Isto sugere que meninos que alcançam a recomendação apresentam 100\% de probabilidade de possuírem o diagnóstico verdadeiramente negativo para a SM, ou seja, estão protegidos da SM.

Quanto a AF de moderada a vigorosa intensidade durante a semana, estes se apresentaram quase $50 \%$ menores frente aos valores medidos nos fins de semana. Estes dados não corroboram outros estudos realizados em crianças da mesma faixa etária em outros países (Comte et al., 2013; Konharn, Santos, \& Ribeiro, 2012). Comte et al. (2013) verificaram AF de moderada a vigorosa intensidade durante a semana foram $30 \%$ maiores. No entanto, o envolvimento em atividades físicas aos fins de semana parece ser fundamental para a saúde, pois o IMC foi inversamente correlacionado ao nível de atividade física de crianças envolvidas em atividades aos fins de semana, principalmente aquelas que têm acesso a áreas exclusivas para tal finalidade como parques e praças (Fairclough, Ridgers, \& Welk, 2012).

\section{Conclusão}

Concluiu-se que o NAF > 3,55 apresenta proteção para doenças cardiovasculares em ambos os sexos. Mais ainda, meninos necessitam de pelo menos 135 minutos de envolvimento em AF de moderada a vigorosa intensidade e 13000 passos por dia para alcançarem resultados positivos à saúde.

\section{Referências}

Activity level and cardiovascular risk (2006). Archives of Disease in Childhood, 91(12), 1017-1017. doi: 10.1136adc.2006.1 10049

Adamo, K. B., Prince, S. A., Tricco, A. C., Connor-Gorber, S., \& Tremblay, M. (2009). A comparison of indirect versus direct measures for assessing physical activity in the pediatric population: a systematic review. International Journal of Pediatric Obesity, 4(1), 2-27. doi: 10.1080/17477160802315010

Adams, M. A., Caparosa, S., Thompson, S., \& Norman, G. J. (2009). Translating physical activity recommendations for overweight adolescents to steps per day. American Journal of Preventive Medicine, 37(2), 137-140. doi: 10.1016/ j.amepre.2009.03.016

Andersen, L. B., Sardinha, L. B., Froberg, K., Riddoch, C. J., Page, A. S., \& Anderssen, S. A. (2008). Fitness, fatness and clustering of cardiovascular risk factors in children from Denmark, Estonia and Portugal: the European Youth Heart Study. International Journal of Pediatric Obesity, 3 Suppl 1, 58-66. doi: 790629714 [pii] 10.1080/17477160801896366 [doi]

Anderson, S., Economos, C., \& Must, A. (2008). Active play and screen time in US children aged 4 to 11 years in relation to sociodemographic and weight status characteristics: a nationally representative cross-sectional analysis. BMC Public Health, 8(1), 366.

Bouchard, C., Tremblay, A., Leblanc, C., Lortie, G., Savard, R., \& Theriault, G. (1983). A method to assess energy expenditure in children and adults. American Journal of Clinical Nutrition, 37(3), 461-467.

Caspersen, C. J., Powell, K. E., \& Christenson, G. M. (1985). Physical Activity, Exercise and Physical Fitness: Definitions and Distinctions for Health - Related Research. Public Health Reports.

Cole, T. J., Bellizzi, M. C., Flegal, K. M., \& Dietz, W. H. (2000). Establishing a standard definition for child overweight and obesity worldwide: international survey. BMJ, 320(7244), 1240-1243.

Colley, R. C., Wong, S. L., Garriguet, D., Janssen, I., Gorber, S. C., \& Tremblay, M. S. (2012). Physical activity, sedentary behaviour and sleep in Canadian children: parent-report versus 
direct measures and relative associations with health risk. Health reports / Statistics Canada, Canadian Centre for Health Information $=$ Rapports sur la santé/Statistique Canada, Centre canadien d'information sur la santé, 23(2), 45.

Comte, M., Hobin, E., Majumdar, S. R., Plotnikoff, R. C., Ball, G. D., \& McGavock, J. (2013). Patterns of weekday and weekend physical activity in youth in 2 Canadian provinces. Applied Physiology, Nutrition Met, 38(2), 115-119. doi: 10.1139/apnm2012-0100

Danielsen, Y. S., Júlíusson, P. B., Nordhus, I. H., Kleiven, M., Meltzer, H. M., Olsson, S. J. G., \& Pallesen, S. (2011). The relationship between life-style and cardio-metabolic risk indicators in children: the importance of screen time. Acta paediatrica (Oslo, Norway : 1992), 100(2), 253.

de Ferranti, S. D., Gauvreau, K., Ludwig, D. S., Neufeld, E. J., Newburger, J. W., \& Rifai, N. (2004). Prevalence of the metabolic syndrome in American adolescents: findings from the Third National Health and Nutrition Examination Survey. Circulation, 110(16), 2494-2497. doi: 01.CIR.0000145117.40114.C7 [pii]10.1161/01.CIR.00001 45117.40114.C7

Department of Health. (2004). At least five a week: evidence on the impact of physical activity and its relationship to health. London, UK: Department of Health.

Eaton, D. K., Kann, L., Kinchen, S., Shanklin, S., Ross, J., Hawkins, J., . . . Wechsler, H. (2010). Youth risk behavior surveillance - United States, 2009. MMWR Surveill Summ, 59(5), 1-142.

Fairclough, S. J., Ridgers, N. D., \& Welk, G. (2012). Correlates of children's moderate and vigorous physical activity during weekdays and weekends. Journal of Physical Activity and Health, 9(1), 129-137.

Farias Júnior, J. C. d., Nahas, M. V., Barros, M. V. G. d., Loch, M. R., Oliveira, E. S. A. d., De Bem, M. F. L., \& Lopes, A. d. S. (2009). Comportamentos de risco à saúde em adolescentes no Sul do Brasil: prevalência e fatores associados. Revista Panamericana de Salud Pública, 25, 344-352.

Freedman, D. S., Patel, D. A., Srinivasan, S. R., Chen, W., Tang, R., Bond, M. G., \& Berenson, G. S. (2008). The contribution of childhood obesity to adult carotid intima-media thickness: the Bogalusa Heart Study. International Journal of Obesity (Lond), 32(5), 749-756. doi: 0803798 [pii]10.1038/ sj.ijo.0803798 [doi]

Froberg, K., \& Andersen, L. B. (2005). Mini review: physical activity and fitness and its relations to cardiovascular disease risk factors in children. International Journal of Obesity (Lond), 29 Suppl 2, S34-39.

Guedes, D. P., \& Guedes, J. E. R. P. (2006). Manual Prático para Avaliação em Educação Física. Barueri-SP.

Harrison, M., Burns, C. F., McGuinness, M., Heslin, J., \& Murphy, N. M. (2006). Influence of a health education intervention on physical activity and screen time in primary school children: 'Switch Off--Get Active'. Journal of Science and Medicine in Sport/Sports Medicine Australia, 9(5), 388.

Haskell, W. L., Lee, I. M., Pate, R. R., Powell, K. E., Blair, S. N., Franklin, B. A., . . . Bauman, A. (2007). Physical activity and public health: updated recommendation for adults from the American College of Sports Medicine and the American Heart Association. Circulation, 116(9), 1081-1093. doi: CIRCULATIONAHA.107.185649 [pii]10.1161/ CIRCULATIONAHA.107.185649

Hughes, A. R., Henderson, A., Ortiz - Rodriguez, V., Artinou, M. L., Reilly, J. J., \& Ortiz-Rodriguez, V. (2006). Habitual physical activity and sedentary behaviour in a clinical sample of obese children.(Pediatric Highlight). International Journal of Obesity, 30(10), 1494.

Hume, C., Salmon, J., Veitch, J., O'Connell, E., Crawford, D., \& Ball, K. (2012). Socio-demographic characteristics of children experiencing socioeconomic disadvantage who meet physical activity and screen- time recommendations: the READI study. Preventive Medicine, 54(1), 61.

King, A. C., Parkinson, K. N., Adamson, A. J., Murray, L., Besson, H., Reilly, J. J., \& Basterfield, L. (2010). Correlates of objectively measured physical activity and sedentary behaviour in English children. The European Journal of Public Health, 21(4), 424-431.

Kong, A., Choi, K.-C., Li, A., Hui, S., Chan, M., Wing, Y., . . . Chan, J. (2010). Association between Physical Activity and Cardiovascular Risk in Chinese Youth Independent of Age and Pubertal Stage. BMC Public Health, 10(1), 303.

Konharn, K., Santos, M. P., \& Ribeiro, J. C. (2012). Differences Between Weekday and Weekend Levels of Moderate-toVigorous Physical Activity in Thai Adolescents. Asia Pacific Journal of Public Health. doi: 10.1177/1010539512459946

Lacobellis, G., Willens, H. J., Barbaro, G., \& Sharma, A. M. (2008). Threshold values of high-risk echocardiographic epicardial fat thickness. [Clinical TrialValidation Studies]. Obesity (Silver Spring), 16(4), 887-892. doi: 10.1038/oby.2008.6

Li, M., Dibley, M. J., Sibbritt, D., \& Yan, H. (2006). Factors associated with adolescents' physical inactivity in Xi'an City, China. Medicine and Science in Sports and Exercise, 38(12), 2075-2085. doi: 10.1249/01.mss.00002338 02.54529.87

Lohman, T. G. (1987). The use of skinfolds to estimate body fatness on children and youth. JOPERD, 58,98-102.

Lohman, T. G., Roche, A. F., \& Martorell, R. (1988). Anthropometric standardization reference manual (Human Kinetics Pub ed.). Champaign: Human Kinetics Pub.

Lwanga, S. K., \& Lemeshow, S. (1991). Sample size determination in health studies: a practical manual. Geneva: World Health Organization.

Ministério da Saúde. (2006). Prevenção clínica de doenças cardiovasculares, cerebrovasculares e renais. Brasília: Ministério da Saúde.

Mushtaq, M. U., Gull, S., Mushtaq, K., Shahid, U., Shad, M. A., \& Akram, J. (2011). Dietary behaviors, physical activity and sedentary lifestyle associated with overweight and obesity, and their socio-demographic correlates, among Pakistani primary school children. The International Journal of Behavioral Nutrition and Physical Activity, 8, 130-130.

Nobre, F. (2010). [Introduction: Brazilian guidelines on hypertension VI. Brazilian Society of Cardiology, Brazilian Society of Hypertension, Brazilian Society of Nephrology]. [Introductory]. Jornal Brasileiro de Nefrologia, 32 Suppl 1, III.

Pate, R. R., O'Neill, J. R., \& Lobelo, F. (2008). The evolving definition of "sedentary". Exercise and Sport Science Review, 36(4), 173-178. doi: 10.1097/JES.0b013e3181877d1a000036 77-200810000-00002 [pii]

Pearce, M. S., Basterfield, L., Mann, K. D., Parkinson, K. N., \& Adamson, A. J. (2012). Early predictors of objectively measured physical activity and sedentary behaviour in 8-10 year old children: the gateshead millennium study. PLOS ONE, 7(6), e37975.

Pulsford, R. M., Cortina-Borja, M., Rich, C., Kinnafick, F. E., Dezateux, C., \& Griffiths, L. J. (2011). Actigraph accelerometer-defined boundaries for sedentary behaviour and 
physical activity intensities in 7 year old children. [Clinical Trial Research Support, Non-U.S. Gov't]. PLoS One, 6(8), e21822. doi: 10.1371/journal.pone.0021822

Schisterman, E. F., Faraggi, D., Reiser, B., \& Trevisan, M. (2001). Statistical inference for the area under the receiver operating characteristic curve in the presence of random measurement error. American Journal of Epidemiology, 154(2), 174-179.

Schusterova, I., Leenen, F. H., Jurko, A., Sabol, F., \& Takacova, J. (2013). Epicardial adipose tissue and cardiometabolic risk factors in overweight and obese children and adolescents. Pediatric Obesity. doi: 10.1111/j.2047-6310.2012.00134.x

Sisson, S. B., Broyles, S. T., Baker, B. L., \& Katzmarzyk, P. T. (2010). Screen Time, Physical Activity, and Overweight in U.S. Youth: National Survey of Children's Health 2003. Journal of Adolescent Health, 47(3), 309-311.

Slaughter, M. H., Lohman, T. G., Boileau, R. A., Horswill, C. A., Stillman, R. J., Van Loan, M. D., \& Bemben, D. A. (1988). Skinfold equations for estimation of body fatness in children and youth. Human Biology, 60(5), 709-723.

Steinberger, J., \& Daniels, S. R. (2003). Obesity, insulin resistance, diabetes, and cardiovascular risk in children: an American Heart Association scientific statement from the Atherosclerosis, Hypertension, and Obesity in the Young Committee (Council on Cardiovascular Disease in the Young) and the Diabetes Committee (Council on Nutrition, Physical Activity, and Metabolism). Circulation, 107(10), 1448-1453.

Tremblay, M. S., Leblanc, A. G., Janssen, I., Kho, M. E., Hicks, A., Murumets, K., . . . Duggan, M. (2011). Canadian sedentary behaviour guidelines for children and youth. [Guideline Research Support, Non-U.S. Gov't Review]. Applied Physiology and Nutrition Metabolism, 36(1), 59-64; 65-71. doi: 10.1139/H11012

Tudor-Locke, C., \& Bassett, D. R., Jr. (2004). How many steps/ day are enough? Preliminary pedometer indices for public health. [Review]. Sports Medicine, 34(1), 1-8.

Tudor-Locke, C., Craig, C., Beets, M., Belton, S., Cardon, G., Duncan, S., . . . Blair, S. (2011). How many steps/day are enough? for children and adolescents. International Journal of Behavioral Nutrition and Physical Activity, 8(1), 78.

Tudor-Locke, C., Washington, T. L., Ainsworth, B. E., \& Troiano, R. P. (2009). Linking the American Time Use Survey (ATUS) and the Compendium of Physical Activities: methods and rationale. Journal of Physical Activity and Health, 6(3), 347353.

U.S. Department of Health and Human Services. (2008). Physical activity guidelines for Americans. Okla Nurse, 53(4), 25.

Vincenti, S. D., \& Pangrazi, R. P. (2002). An examination of the activity patterns of elementary school children. Pediatrics Exercise Science, 4(14), 434-441.

Weghuber, D., Zelzer, S., Stelzer, I., Paulmichl, K., Kammerhofer, D., Schnedl, W., . . . Mangge, H. (2013). High Risk vs. "Metabolically Healthy" Phenotype in Juvenile Obesity Neck Subcutaneous Adipose Tissue and Serum Uric Acid are Clinically Relevant. Experimental and Clinical Endocrinology and Diabetes. doi: 10.1055/s-0033-1341440

WHO. (1995). Committee on Physical Status: the use and interpretation of anthropometry. Geneva: World Health Organization,.

WHO. (2000). Obesity: preventing and managing the global epidemic. Report of a WHO consultation. World Health Organ Tech Rep Ser, 894, 1-253.

World Health Organization. (1985). Energy and protein requirements. Report of a joint FAO/WHO/UNU Expert Consultation. World Health Organ Tech Rep Ser, 724, 1-206.
World Health Organization. (2010). Global recommendations on physical activity for health. Geneva: World Health Organization,

\section{Nota dos autores}

Alynne Christian Ribeiro Andaki e Adelson Luiz Araújo Tinoco são membors do Programa de Pós-graduação em Ciência da Nutrição da Universidade Federal de Viçosa-UFV, Viçosa, MG.

Roberto Andaki Júnior é membro do Departamento de Ciências do Esporte, Universidade Federal do Triângulo Mineiro-UFTM, Uberaba, MG..

Amanda Santos e Edmar Lacerda Mendes são membors do Programa de Mestrado em Educação Física da Universidade Federal do Triângulo Mineiro-UFTM, Uberaba, MG.

Ciro José Britoé membro do Núcleo de Estudos em Desempenho Esportivo e Saúde da Universidade Federal de Sergipe, Aracaju, SE

\section{Correspondência}

Alynne Christian Ribeiro Andaki

Rua Virmondes Roel da Silva, n 18. Bairro Jardim Nenê Gomes. Uberaba/ MG

Telefone: (34) 84187947

Email: alynneandaki@yahoo.com.br ou alynne@ufv.br

O presente estudo é resultado de dissertação de mestrado.

Artigo completo de trabalho apresentado no VIII Congresso Internacional de Educação Física e Motricidade Humana e XIV Simpósio Paulista de Educação Física.

Declaração de Conflito de Interesses: Os autores declararam ausência de conflitos de interesse no que diz respeito à pesquisa, autoria e/ou publicação deste artigo.

Manuscrito recebido em 30 de março de 2013

Manuscrito aceito em 10 de maio de 2013 